\title{
NOUVELLe
}

Les fonctions innées des lymphocytes T CD8 dans la lutte contre le cancer

Alice Barbarin ${ }^{1,2}$, André Herbelin ${ }^{1,2}$, Jean-Marc Gombert ${ }^{1,2}$
${ }^{1}$ Inserm U1082, Université de Poitiers - UFR Faculté de sciences fondamentales et appliquées (SFA), Pôle biologie santé (PBS) B36, 1, rue Georges Bonnet - TSA 51106, 86073 Poitiers Cedex 9 , France.

${ }^{2} \mathrm{CHU}$ de Poitiers, 2, rue de la Milétrie, 86000 Poitiers, France. andre.herbelin@inserm.fr jean-marc.gombert@chu-poitiers.fr
Une nouvelle classe de lymphocytes T à mémoire non-conventionnelle : les cellules T CD ${ }^{+}$innées

Les cellules immunitaires sont habituellement regroupées en deux catégories: celles appartenant à l'immunité adaptative (les lymphocytes $T$ et $B$ ) et celles appartenant à l'immunité innée (les macrophages, les cellules NK pour «natural killer», les monocytes, etc.). Les premières expriment leur récepteur spécifique d'un antigène, le TCR ( $T$ cell receptor) pour les lymphocytes $T$, ou le $B C R$ ( $B$ cell receptor) pour les lymphocytes $B$, par réarrangement de gènes, et elles ont la capacité de mémoriser les antigènes déjà rencontrés. Les secondes expriment en revanche des récepteurs conservés qui lient des motifs moléculaires associés aux pathogènes (PAMP) ou aux dégâts cellulaires (DAMP). Depuis environ vingt ans, cette vue du système immunitaire s'est révélée plus complexe avec l'identification de lymphocytes $T \alpha \beta$ (phénotype attribué aux lymphocytes participant à l'immunité adaptative, décrivant les deux sous-unités du TCR, $\alpha$ et $\beta$ ) qui présentent des caractéristiques de cellules innées: une reconnaissance de motifs moléculaires particuliers, un délai de réponse court, une production de cytokines et des fonctions effectrices indépendantes du TCR. Ces populations immuno-régulatrices interviennent en amont de la réponse adaptative, dont elles déterminent la qualité et la robustesse. Leur rôle protecteur dans l'immuno-surveillance des cancers a aussi été démontré [1]. L'archétype de ces lymphocytes $T$ dits non-conventionnels sont les cellules iNKT, pour «invariant natural killer $T$ cells », découvertes au début des années 1990. Elles possèdent un TCR semi-invariant reconnaissant des antigènes glycolipidiques présentés par la molécule non classique du complexe majeur d'histocompatibilité ( $C M H)$ de classe I, CDId. Depuis la découverte des iNKT, de nouveaux membres de la famille des lymphocytes T non-conventionnels sont régulièrement identifiés [1]. C'est dans ce contexte, qu'au début des années 2000, dans plusieurs modèles murins, une nouvelle population de lymphocytes $T$ non-conventionnels a été découverte: les lymphocytes $T$ $\mathrm{CD}^{+}$« inné-mémoire ». Bien que n'ayant jamais rencontré d'antigènes, ces cellules ont pourtant des caractéristiques de cellules mémoires qui se traduisent, notamment, par la présence d'une signature fonctionnelle: la forte expression du facteur de transcription Eomesodermin (Eomes) $[2,3]$. Dans le modèle murin, on distingue les lymphocytes $T$ $\mathrm{CD}^{+}$mémoire «inné », d'origine thymique, des lymphocytes T CD $8^{+}$mémoire «virtuel», qui se développent en périphérie, grâce à l'expression à la surface de leur membrane de CD49d, sous-unité $\alpha 4$ d'une intégrine. Le développement de la population de lymphocytes T CD8 ${ }^{+}$ « inné-mémoire » est sous le contrôle de plusieurs cytokines : I'interféron- $\alpha$ (IFN$\alpha)$ et les interleukines 4 (IL-4) et 15 $($ IL-15) [4] $(\rightarrow)$.

$(\rightarrow)$ Voir la Nouvelle de V. Martinet et S. Goriely, $m / s n^{\circ} 3$, Chez l'homme, mars 2016, page 236 l'équivalent des lymphocytes T CD $8^{+}$ « inné-mémoire » murins n'est pas encore clairement caractérisé et l'étude de leur développement, dans un envi- ronnement dépourvu d'antigène, est difficile à appréhender. Cependant, plusieurs équipes ont identifié des cellules $\mathrm{T} C D 8^{+}$ayant des caractéristiques innées, reflétées par l'expression de récepteurs habituellement exprimés par les cellules NK: CD56, KIR, NKG2A, NKG2C et CD94 ${ }^{l}$, d'où leur appellation de cellules $\mathrm{TCD}^{+}$de type NK (NK-like CD8 ${ }^{+} \mathrm{T}$ cells). Ces cellules présentent un phénotype de lymphocyte $T$ effecteur terminal ( $T_{\varepsilon M R A}$, pour mémoire effectrice exprimant le CD45RA) et elles sécrètent la cytokine immuno-stimulatrice interféron- $\gamma$ (IFN$\gamma$ ) en réponse aux interleukines 12 et 18 (IL-12 et IL-18), et des messagers de la réponse inflammatoire [5]. En raisonnant par analogie avec le modèle murin, nous avons nous-mêmes identifié, chez l'homme adulte, des lymphocytes T CD $8^{+}$, qui expriment les récepteurs KIR/NKG2A, avec une forte expression du facteur de transcription Eomes, et qui répondent à une stimulation par les IL-12 et IL-18 [6]. Ces cellules sont aussi présentes dans le sang de cordon, une source accessible de cellules qui sont considérées comme une approximation des cellules thymiques chez l'homme. Ainsi, pour la première fois, nous associons les marqueurs de type NK, les fonctions de type inné, et la fonction mémoire reflétée par l'expression d'Eomes, sur une population de lymphocytes T $C D 8^{+}$ que nous avons dénommée T $C D 8^{+}$innés (innate $C D 8^{+} T$ cells). Plus récemment, nous avons montré que CD49d, un mar-

\footnotetext{
${ }^{1}$ CD56 : molécule d'adhérence des cellules NK et des lymphocytes T; KIR : récepteurs activateur ou inhibiteur des cellules NK appartenant à la superfamille des immunoglobulines; NKG2A/
} NKG2C/CD94 : récepteurs de type lectine des cellules NK. 


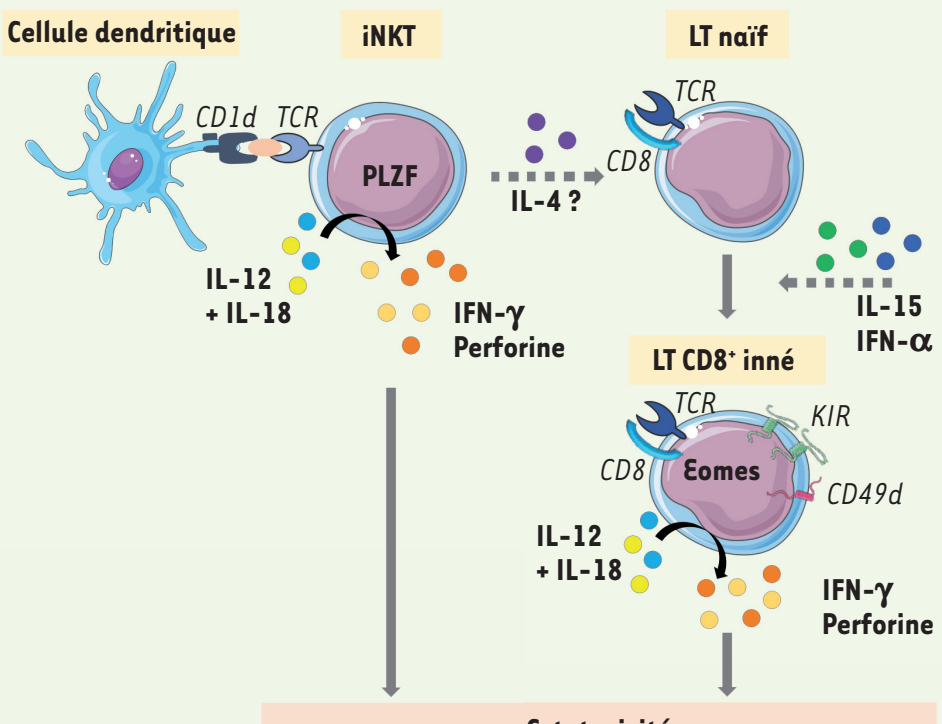

Cytotoxicité
Figure 1. L'axe iNKT/T CD8+ innés dans la cytotoxicité anti-tumorale. Les cellules iNKT sont définies par leur TCR semi-invariant reconnaissant les antigènes présentés par la molécule non classique du CMH de classe I, CDld, exprimée par les cellules dendritiques. Elles expriment fortement le facteur de transcription PLZF (promyelocytic leukemia zinc-finger) et peuvent sécréter l'interleukine 4 (IL-4), une interleukine probablement nécessaire au développement des cellules TCD ${ }^{+}$innées. Ces cellules sont caractérisées par un TCR de type $\alpha \beta$ et expriment des récepteurs de surface KIR et CD49d (sous-unité $\alpha 4$ d'une intégrine), ainsi que le facteur de transcription Eomes. En réponse aux cytokines inflammatoires IL-12 et IL-18, les iNKT et les T CD $8^{+}$innés ont la capacité de sécréter l'IFN- $\gamma$ et la perforine, des molécules cytotoxiques essentielles pour lutter contre les tumeurs. CDId : molécule non classique du complexe majeur d'histocompatibilité de classe I; IFN : interféron; IL : interleukine ; iNKT: invariant natural killer T; KIR : récepteurs activateur ou inhibiteur des cellules NK de type immunoglobuline; LT : lymphocyte T; TCR : T cell receptor.

queur discriminant des lymphocytes $T$ CD8 ${ }^{+}$«inné-mémoire » chez la souris, est préférentiellement exprimé par cette population, et que son expression peut être corrélée plus particulièrement avec la fonction de sécrétion d'IFN- $\gamma$ en réponse à la stimulation par les $\mathrm{IL}-12$ et IL-18 [5]. Chez l'homme, les mécanismes et les molécules impliqués dans la différenciation des lymphocytes T CD ${ }^{+}$ innés ne sont pas ou peu connus. Cependant, dans certains modèles murins, le développement de cette population semble dépendre de I'IL-4 produite par des cellules iNKT exprimant fortement le facteur de transcription PLZF (promyelocytic leukemia zinc-finger), qui est une signature fonctionnelle de ces cellules. Nos résultats sont en faveur de cette hypothèse soutenue par l'existence d'une corrélation positive entre l'expression d'Eomes dans les lymphocytes T CD $8^{+}$innés et celle de PLZF dans les cellules iNKT ainsi que par l'effet prolifératif/homéostatique direct de I'IL-4 in vitro sur les T CD8 ${ }^{+}$innés $[6,7]$.

\section{Fonction des $\mathrm{T} \mathrm{CD8}{ }^{+}$innés :}

la surveillance immunitaire

des cancers?

Jusqu'à présent, une seule série de travaux, réalisés chez la souris, s'est intéressée à la fonction des lymphocytes T CD8 inné-mémoire en démontrant leur rôle protecteur contre certaines infections $[8,9]$. Or, l'arsenal cytotoxique, dont disposent les lymphocytes $\mathrm{T} C D 8^{+}$innés (la perforine, le granzyme B et l'IFN- $\gamma$ ), est aussi compatible avec des fonctions anti-tumorales. De plus, connaissant le possible lien entre les iNKT et les lymphocytes $\mathrm{T} \mathrm{CD8}^{+}$innés, et en prenant en compte nos précédents travaux montrant que les iNKT sont déficientes dans la leucémie myéloïde chronique (LMC), une hémopathie maligne [10], nous avons logiquement poursuivi cette étude en nous focalisant sur les lymphocytes $T$ $\mathrm{CD}^{+}$innés. Nous démontrons ainsi pour la première fois, que la population $T \mathrm{CD} 8^{+}$ innée est affectée quantitativement et qualitativement, lors de la phase chronique de la LMC, et qu'elle peut être en partie rétablie chez les patients en rémission [7]. Ces travaux nous ont permis de proposer un scénario pour cette maladie ayant pour origine l'expression de l'oncogène BCR-ABL (breakpoint cluster region-abelson) dans la lignée myéloïde [5]. En effet, nous montrons qu'une des conséquences de l'expression de BCR-ABL par les cellules dendritiques présentatrices d'antigènes est l'absence de la molécule CDld à leur surface [11]. Or, ce mécanisme pourrait conduire à la diminution numérique et fonctionnelle des iNKT que nous observons au cours de cette maladie, en particulier la diminution de l'expression de PLZF et de la sécrétion d'IL-4 [10], d'où l'impact sur le développement des lymphocytes T CD8 ${ }^{+}$ innés. Ces travaux portant sur la LMC montrent le lien entre les cellules iNKT et les lymphocytes $T$ CD8 ${ }^{+}$innés, et nous permettent d'émettre l'hypothèse d'un axe iNKT/T CD8 ${ }^{+}$innés dont l'un des rôles pourrait être une activité anti-tumorale directe via leur capacité de production de molécules cytotoxiques (Figure 1).

En parallèle des études visant à démontrer la fonction anti-tumorale des lymphocytes T CD8 ${ }^{+}$innés, nous avons élargi nos recherches sur les lymphocytes $T$ $\mathrm{CD}^{+}$innés à deux cancers solides: le cancer du sein et le cancer de l'ovaire. Nous avons ainsi pu montrer la présence des lymphocytes $T$ CD $8^{+}$innés au sein de la tumeur principale et, dans le cas 
du cancer de l'ovaire, la présence de cellules iNKT, avec également une corrélation positive entre l'expression de PLZF, par les iNKT, et celle d'Eomes, par les T CD8 ${ }^{+}$innés [5]. La présence de ces deux types cellulaires dans les tumeurs, dénote un probable recrutement de ces cellules, mais il reste à élucider si ces cellules ont un effet bénéfique ou délétère dans ces tumeurs solides.

Il convient enfin de soulever la question des phénomènes d'immuno-subversion des tumeurs et, en particulier, celui du blocage immunitaire possible des lymphocytes T CD8 ${ }^{+}$innés par les cellules tumorales. En effet, à l'heure des premiers succès des immunothérapies visant à réveiller le système immunitaire du patient pour qu'il lutte lui-même contre la tumeur, il appa- raît nécessaire de déterminer si les lymphocytes T CD8 ${ }^{+}$innés peuvent être une cible potentielle de ces nouvelles stratégies. $\diamond$

The CD8* T cell innate function

in the war against cancer

\section{LIENS D'INTÉRÊT}

Les auteurs déclarent n'avoir aucun lien d'intérêt concernant les données publiées dans cet article.

\section{RÉFÉRENCES}

1. Godfrey DI, Uldrich AP, McCluskey J, et al. The burgeoning family of unconventional T cells. Nat Immunol 2015; 16 : 1114-23.

2. Jameson SC, Lee YJ, Hogquist KA. Innate memory $T$ cells. Adv Immunol 2015 ; 126 : 173-213.

3. Lee YJ, Jameson SC, Hogquist KA. Alternative memory in the CD8 T cell lineage. Trends Immunol 2011 ; 32 : 50-6.

4. Martinet $V$, Goriely S. La mémoire virtuelle des lymphocytes T cytotoxiques. Med Sci (Paris) 2016 ; $32: 236-8$.
5. Barbarin A, Cayssials $\varepsilon$, Jacomet F, et al. Phenotype of NK-like $\operatorname{CD} 8^{+} T$ cells with innate features in humans and their relevance in cancer diseases. Front Immunol $2017 ; 8: 316$.

6. Jacomet F, Cayssials $\varepsilon$, Basbous $S$, et al. Evidence for eomesodermin-expressing innate-like CD8 ${ }^{+} \mathrm{KIR} /$ NKG2A ${ }^{+} T$ cells in human adults and cord blood samples. Eur J Immunol 2015 ; 45 : 1926-33.

7. Jacomet F, Cayssials $\varepsilon$, Barbarin A, et al. The hypothesis of the human iNKT/innate $C D 8^{+} T$-cell axis applied to cancer: evidence for a deficiency in chronic myeloid leukemia. Front Immunol 2016; $7: 688$.

8. Renkema KR, Li G, Wu A, et al. Two separate defects affecting true naive or virtual memory $T$ cell precursors combine to reduce naive $T$ cell responses with aging. J Immunol 19502014 ; 192 : 151-9.

9. Lee A, Park SP, Park CH, et al. IL-4 induced innate $\mathrm{CD}^{+} \mathrm{T}$ cells control persistent viral infection. PLOS Pathog 2015 ; 11 : el005193.

10. Rossignol A, Levescot A, Jacomet F, et al. Evidence for $B C R-A B L-$ dependent dysfunctions of iNKT cells from chronic myeloid leukemia patients. Eur J Immunol $2012 ; 42: 1870-5$

11. Basbous S, Levescot A, Piccirilli N, et al. The Rho-ROCK pathway as a new pathological mechanism of innate immune subversion in chronic myeloid leukaemia.J Pathol 2016 ; $240: 262-8$.

\section{NOUVELLE}

\section{Vers de nouveaux marqueurs
pronostiques dans
les leucémies aiguës myéloiides \\ Analyse génomique des altérations du nombre de copies}

Fanny Gonzales, Meyling Cheok

> La leucémie aiguë myéloïde (LAM) est une hémopathie maligne caractérisée par une prolifération anarchique de précurseurs myéloïdes, appelés blastes. Il s'agit d'une entité très hétérogène, sur le plan clinique d'abord, avec une grande variabilité inter-individuelle des symptômes initiaux et de la réponse aux thérapeutiques. La survie à 5 ans varie de $10 \%$ chez les sujets âgés à $60 \%$ chez l'enfant et le jeune adulte [1]. L'hétérogénéité se manifeste également au niveau biologique, avec huit sous-types cytologiques et des caractéristiques immuno-phénotypiques, cytogénétiques et moléculaires différentes selon les patients [2]. Malgré cette hétérogénéité, le traitement par chimiothérapie, à base d'anthracyclines et de cytarabine, reste identique chez tous les patients. II est souvent complété par une allogreffe de cellules souches hématopoïétiques (CSH).

Actuellement, la prise en charge thérapeutique, fondée sur une stratification pronostique en trois groupes de risque (favorable, défavorable ou intermédiaire, ce dernier regroupant les $2 / 3$ des patients), repose presque exclusivement sur les anomalies cytogénétiques [2]. Cette classification reste cependant imparfaite puisque la réponse aux traitements et le pronostic varient au sein d'un même groupe de risque.
Univ. Lille, Inserm, CHU Lille, UMR-S 1172 - JPArc-Centre de Recherche Jean-Pierre Aubert Neurosciences et Cancer, F-59000 Lille, France.

fanny.gonzales@inserm.fr

Afin d'améliorer la prise en charge des patients, il apparaît essentiel de trouver de nouveaux marqueurs pronostiques. Dans cette optique, notre équipe s'est intéressée aux altérations du nombre de copies (CNA, copy number alteration) comme potentiels marqueurs pronostiques [3].

Les cellules cancéreuses sont caractérisées par l'accumulation et la combinaison de multiples anomalies génétiques et épigénétiques $[4,5](\rightarrow)$.

$(\rightarrow)$ Voir la Synthèse de F. Damm et al., $\mathrm{m} / \mathrm{s} \mathrm{n}^{\circ}$ 5, mai 2012, page 449

Celles-ci pourraient constituer des marqueurs pronostiques facilement identifiables, comme c'est le cas pour 